\title{
Sex Differences in Characteristics Associated with Potentially Inappropriate Medication Use and Associations with Functional Capacity in Older Participants of the Berlin Aging Study II
}

\author{
Sarah Toepfer ${ }^{a}$ Maximilian König ${ }^{a}$ Dominik Spira $^{a} \quad$ Johanna Drewelies $^{b}$ \\ Reinhold Kreutz ${ }^{c}$ Juliane Bolbrinker ${ }^{c} \quad \| j a$ Demuth $^{a, d}$ \\ ${ }^{a}$ Charité - Universitätsmedizin Berlin, corporate member of Freie Universität Berlin and Humboldt-Universität zu \\ Berlin, Lipid Clinic at the Interdisciplinary Metabolism Center, Berlin, Germany; ${ }^{b}$ Humboldt-Universität zu Berlin, \\ Department of Psychology, Berlin, Germany; ${ }^{c}$ Charité - Universitätsmedizin Berlin, corporate member of Freie \\ Universität Berlin and Humboldt-Universität zu Berlin, Institute of Clinical Pharmacology and Toxicology, Berlin,

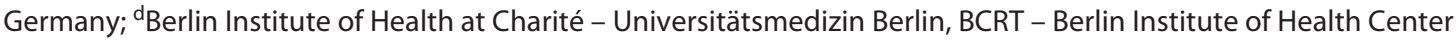 \\ for Regenerative Therapies, Berlin, Germany
}

\section{Keywords}

Inappropriate prescribing $\cdot$ EU(7)-PIM $\cdot$ Berlin Aging

Study II · Education · Frailty · Falls $\cdot$ Depression

\begin{abstract}
Introduction: Medication safety is a vital aim in older adults' pharmacotherapy. Increased morbidity and vulnerability require particularly careful prescribing. Beneath avoiding unnecessary polypharmacy and prescribing omissions, physicians have to be aware of potentially inappropriate medications (PIMs) and related outcomes to optimize older adults' drug therapy, and to reduce adverse drug events. Objective: The aim of this study was to identify participants characteristics associated with PIM use and associations of PIM use with functional capacity with a focus on sex differences. Methods: Multivariable logistic regression analyses of crosssectional Berlin Aging Study II (BASE-II) data ( $N=1,382$, median age 69 years, interquartile range $67-71,51.3 \%$ women) were performed with PIM classification according to the EU(7)-PIM list. Results: In the overall study population, higher education was associated with lower odds of PIM use
\end{abstract}

karger@karger.com www.karger.com/ger

Karger $\stackrel{\text { ' }}{5}$

BOPEN ACCESS
(C) 2021 The Author(s)

Published by S. Karger AG, Basel

This is an Open Access article licensed under the Creative Common Attribution-NonCommercial-4.0 International License (CC BY-NC) (http://www.karger.com/Services/OpenAccessLicense), applicable to the online version of the article only. Usage and distribution for commercial purposes requires written permission. (odds ratio [OR] 0.93, confidence interval [Cl] 95\% 0.87-0.99, $p=0.017$ ). Falls (OR 1.53, Cl 95\% 1.08-2.17, $p=0.016$ ), frailty/ prefrailty (OR 1.68, 1.17-2.41, $p=0.005)$, and depression (OR $2.12, \mathrm{Cl} 95 \% 1.32-3.41, p=0.002$ ) were associated with increased odds of PIM use. A better nutritional status was associated with lower odds of PIM use (OR 0.88, CI 95\% 0.81$0.97, p=0.008$ ). In the sex-stratified analysis, higher education was associated with lower odds of PIM use in men (OR $0.90, \mathrm{Cl} 95 \% 0.82-0.99, p=0.032$ ). Frailty/prefrailty was associated with increased odds of PIM use in men (OR 2.04, Cl $95 \% 1.18-3.54, p=0.011$ ) and a better nutritional status was associated with lower odds of PIM use in men (OR $0.83, \mathrm{CI}$ $95 \% 0.72-0.96, p=0.011$ ). Falls in the past 12 months were related to an increased prevalence of PIM use in women (OR $1.74, \mathrm{Cl} 95 \% 1.10-2.75, p=0.019)$. Depression was associated with a higher prevalence of PIM use in both men (OR $2.74, \mathrm{CI}$ 95\% 1.20-6.24, $p=0.016$ ) and women (OR 2.06, Cl 95\% 1.14$3.71, p=0.017)$. We did not detect sex differences regarding the overall use of drugs with anticholinergic effects, but more men than women used PIMs referring to the cardiovas-

Juliane Bolbrinker and Ilja Demuth share last authorship.
Correspondence to:

Ilja Demuth, ilja.demuth@ charite.de 
cular system $(p=0.036)$, while more women than men used PIMs referring to the genitourinary system and sex hormones $(p<0.001)$. Conclusion: We found similarities, but also differences between men and women as to the associations between PIM use and participants' characteristics and functional capacity assessments. The association of lower education with PIM use may suggest that physicians' prescribing behavior is modified by patient education, a relationship that could evolve from more critical attitudes of educated patients towards medication use. We conclude that sex differences in associations of PIM use with functional capacities might be partly attributable to sex differences in drug classes used, but not with regard to anticholinergics, as these are used to a similar extent in men and women in the cohort studied here.

(c) 2021 The Author(s)

Published by S. Karger AG, Basel

\section{Introduction}

Improving medication safety is of particular importance in older adults' drug therapy. To evaluate medication quality and to improve medication safety, it is necessary to be aware of suboptimal prescribing, including unnecessary polypharmacy, potentially inappropriate medications (PIMs), and underuse of required drugs. Insufficient individualization of drug therapy concerning age-related changes, like decline in renal function, decreasing muscle mass, and altered drug sensitivity results particularly from underrepresentation of geriatric patients in clinical trials. This lack of individualization leads to frequent prescribing of PIMs in old age, unnecessary polypharmacy, and unnecessary exposure to medications with doubtful efficacy [1]. Drugs are denoted to be PIMs if their potential harm outweighs the expected clinical benefit [2]. As stated in a recently published narrative literature review, PIM use is not only associated with specific patient characteristics like lower socioeconomic status but is also related to inadequate care management such as having several prescribers, frequent physician visits, and longer stays in nursing homes or hospitals [1].

Among numerous national and international PIM lists, the EU(7)-PIM list is a comprehensive, explicit, sensitive, and current tool that classifies 282 drugs to be inappropriate for people aged 65 years and older $[3,4]$. A study investigating the applicability of EU(7)-PIM criteria in cross-national studies in European countries reported PIMs from the EU(7)-PIM list to be approved for clinical use in Europe more frequently than those listed

Associations with Inappropriate

Medication Use in Older Participants in the Beers list 2015 [5]. Thus, for PIM assessment in European studies, the EU(7)-PIM list appears to be the most suitable instrument. Furthermore, morbidity has recently been associated with PIM use based on the EU(7)-PIM list but not based on the German national PRISCUS list [6] in the Berlin Aging Study II (BASE-II) cohort [7], indicating an additional benefit of the EU(7)PIM list to trace negative outcomes.

Additionally, previous analyses showed that PIM use is more prevalent in women and is associated with a higher number of regular medications in the BASE-II cohort [7]. We identified a total number of 402 PIMs (EU[7]PIM list) in 313 participants (22.6\%) in our previous study [7]. Independent of the applied list to assess drug inappropriateness, being female is an established risk factor for PIM use [8-11]. For example, the prevalence of depression, anxiety disorders, and sleep disorders is higher in women than in men, while drugs that are classified as PIMs are typically used for these indications [12]. Little is known about sex differences in further characteristics related to PIM use, such as education or lifestyle factors. Both lower and higher prevalence of PIM use in relation to lifestyle factors or education is conceivable. A study investigating modifiable factors related to PIM use in patients in primary care found alcohol consumption and current smoking as components of "unhealthy behaviors" increasing the odds for PIM use [13]. Although polypharmacy and regular visits to a physician were also defined as "unhealthy behaviors" in this investigation, which can be rated critically, the idea to trace modifiable factors for PIM use appears worthwhile. As reported in a systematic review in 2019, the most common statistically significant factors associated with PIM use were polypharmacy, female sex, and frequent utilization of medical services [14].

A recently published German study examined PIM prescribing on the basis of the EU(7)-PIM list in 6.0 million individuals at least 65 years old. A decreasing prevalence of PIM prescribing from $56.9 \%$ in 2009 to $45.1 \%$ in 2019, a higher prevalence of PIM prescribing in women, and an association between the total number of prescribed drugs and PIM prescription was reported [15]. The ESTHER study (German name: Epidemiologische Studie zu Chancen der Verhütung, Früherkennung und optimierten THerapie chronischer ERkrankungen in der älteren Bevölkerung) examined the prevalence of PIM use defined by the EU(7)-PIM list, related characteristics, and outcomes in a large community-dwelling German cohort [9]. Therefore, the ESTHER cohort is an important comparative population to evaluate and to discuss 
our findings $[9,16]$. This study identified female sex and an increased BMI (body mass index) to be related to PIM use [9].

Cognitive impairment, frailty, morbidity, and functional impairment have been described to be associated with PIM use in cross-sectional analysis [9]. By comparing different PIM lists, such as the PRISCUS list [6], the EU(7)-PIM list [2], the Beers list 2015 [17], and the Beers dementia sub-list (as part of the Beers list 2015), including anticholinergics to be avoided in patients with dementia, the strongest association of PIM use and frailty was described for the Beers dementia sub-list [16]. Based on this result, it seems possible that PIMs differ in their impact on the outcomes studied. Furthermore, sex differences with regard to negative PIM effects are conceivable, for example, due to differences in drug sensitivity, which has already been described for digitalis glycosides [18].

Little is known about sex differences regarding associations of PIM use with lifestyle and sociodemographic factors, functional capacity, and the sex-dependent distribution of PIMs in different drug classes. To address this gap, we carried out the following analyses. The objective of this study was to focus on participants' characteristics and functional assessment results related to PIM use according to the EU(7)-PIM list [2] and the sex-dependent distribution of users within therapeutic groups based on the Anatomical Therapeutic Chemical (ATC) classification system [19].

\section{Methods}

\section{BASE-II Study Population}

Cross-sectional baseline data from the BASE-II were used for the current analyses. The BASE-II study has been described in detail before [20,21]. Overall, 2,171 study participants, thereof a group of community-dwelling elderly people (60-84 years old, about $75 \%$ of the study population), and a control group of younger participants (20-36 years old) were comprehensively examined. All participants gave written informed consent. The Ethics Committee of the Charité - Universitätsmedizin Berlin approved the BASE-II study (approval number EA2/029/09).

As part of the study protocol, physicians performed a structured survey, including a history of medication use considering prescribed drugs as well as nonprescribed drugs, and dietary supplements. For the current study, we included 1,382 BASE-II participants (median age 69 years, interquartile range $67-71,51.3 \%$ women), who were at least 65 years old at baseline assessments (2009-2014) because PIM lists are applicable from the age of 65 years onward. We considered all present prescribed and nonprescribed medications and supplements.

\section{PIM Use and Drug Classification}

Drugs were classified as inappropriate according to the EU(7)PIM list [2]. Information about anticholinergic side effects also derived from the EU(7)-PIM list. The classification of PIMs was based on the Anatomical Therapeutic Chemical (ATC) classification system 1st level group (WHO Collaborating Center for Drug Statistics) [19].

\section{Participants' Characteristics Related to PIM Use}

The following characteristics were examined for their association with PIM use: BMI $\left(\mathrm{kg} / \mathrm{m}^{2}\right)$, current smoking (yes/no), alcohol consumption (yes/no), years of education, and living alone (yes/no). The BMI was calculated from measurements of height (m) and weight (kg). Current smoking, alcohol consumption, and living alone were assessed as the self-reported answers to the questions "Are you currently smoking?", "Do you sometimes drink alcohol?", and "Do you live alone?", respectively. Smoking and alcohol consumption were included as dichotomous variables in the analyses to reflect the underlying lifestyle decisions either to smoke or to drink alcohol or not to do so. We captured years of education as the self-reported number of years in formal schooling. These analyses served to identify particular participants' characteristics as potential confounders for the analyses of the relationship between PIM use and results of the functional assessments as described in section 2.4. Morbidity was assessed by a morbidity index largely based on the categories of the Charlson index (see supplement for additional information) [22, 23]. Online supplementary Table 1 (see www.karger.com/doi/10.1159/000518411 for all online suppl. material) shows differences in participants' characteristics between PIM users and non-PIM users.

\section{Functional Assessments and Associations with PIM Use}

We considered results of the following functional geriatric assessments for their association with PIM use: Barthel Index (Activities of Daily Living Scale, ADL), the Lawton/Brody Index (Instrumental Activities of Daily Living Scale, IADL), Tinetti mobility test, Timed “Up \& Go" test (TUG), walking speed test (4 m), handgrip strength, number of self-reported falls within the past 12 months, Mini-Nutritional Assessment (MNA), Mini-Mental State Examination (MMSE), Digit-Symbol Substitution test (DSST), clock drawing test, Center for Epidemiological Studies Depression Scale (CES-D Scale), and a frailty score (Fried) [24-32]. Detailed information on these assessments and their use in the current study is available in the online supplementary material. The assessment characteristics of the overall study population are summarized in online supplementary Table 2.

\section{Statistics}

Calculations were performed with IBM SPSS Statistics, version 25. We used the Mann-Whitney $U$ test to analyze non-normally distributed, continuous variables. The $\chi^{2}$ test was used to detect group differences.

First, we used multivariable logistic regressions to estimate the odds of PIM use for participant characteristics. We included age as a demographic variable, number of regular medications, and morbidity index as variables with confirmed associations with PIM use [7], BMI, current smoking, alcohol consumption, years of education, and living alone as characteristics of interest. Second, to assess the odds of PIM use depending on functional assessment results, we performed multivariable logistic regression analyses for 
Table 1. Sex-stratified results of multivariable logistic regression analysis regarding participants' characteristics related to PIM use

\begin{tabular}{llll}
\hline Participants' characteristics & OR & CI 95\% & $p$ value \\
\hline Men $(N=430)$ & & & \\
BMI (kg/m²) & 1.02 & $0.95-1.10$ & 0.558 \\
$\quad$ Current smoking (yes/no) & 1.46 & $0.64-3.35$ & 0.369 \\
$\quad$ Alcohol consumption (yes/no) & 0.91 & $0.37-2.25$ & 0.845 \\
$\quad$ Years of education & 0.90 & $0.82-0.99$ & 0.032 \\
$\quad$ Living alone (yes/no) & 0.78 & $0.40-1.54$ & 0.477 \\
Women $(N=435)$ & & & \\
BMI (kg/m $\left.{ }^{2}\right)$ & 1.03 & $0.97-1.90$ & 0.313 \\
Current smoking (yes/no) & 0.90 & $0.30-2.40$ & 0.759 \\
$\quad$ Alcohol consumption (yes/no) & 1.50 & $0.67-3.36$ & 0.328 \\
$\quad$ Years of education & 0.95 & $0.87-1.03$ & 0.218 \\
$\quad$ Living alone (yes/no) & 0.85 & $0.53-1.36$ & 0.496 \\
\hline
\end{tabular}

$N=865$. Smoking, alcohol consumption, and living alone were included as binary variables. BMI and years of education were included as continuous variables. BMI, body mass index; OR, odds ratio; $\mathrm{CI}$, confidence interval; PIMs, potentially inappropriate medications. ${ }^{*} p<0.05$.

each functional test separately as we assumed that functional assessment results are independent from each other. We included age, number of regular medications, and morbidity index as covariates with confirmed associations with PIM use [7], as well as years of education which was identified as a variable related to PIM use in the current study.

After analyzing the overall dataset, we stratified the data for sex in order to detect sex differences in the associations found. We were interested in associations between PIM use and functional assessment results that (a) can be confirmed in both the overall and in the sex-stratified analysis or (b) are found in the overall analysis but not in the sex-stratified analysis or (c) are not found in the overall analysis but in the sex-stratified analysis. Statistical significance was established a priori at $p<0.05$.

\section{Results}

Participants' Characteristics Related to PIM Use in the Overall Study Population and Stratified by Sex

Due to missing data on covariates, the number of participants to be included in the logistic regression model assessing the relationship between participants' characteristics and PIM use decreased from 1,382 to 865 (online suppl. Table 3 ). The participants included did not differ significantly with respect to the distribution of sex, morbidity, and education when compared to the subjects excluded because of missing data. Age, however, was slightly higher in those excluded due to missing data when compared to the subjects analyzed here $(p=0.011$, online suppl. Table 3).

Overall, we found education to be associated with lower odds of PIM use (odds ratio [OR] 0.93, confidence interval [CI] 95\% 0.87-0.99, $p=0.017$ ). BMI, current smoking, alcohol consumption, and living alone did not affect the chance of PIM use in the overall study population (online suppl. Table 4).

Because of missing data on covariates, we included 430 of 673 men and 435 of 709 women in the sex-stratified logistic regression models (Table 1). Sex-stratified analyses revealed that longer education time was associated with lower odds of PIM use in men (OR 0.90, CI 95\% $0.82-0.99, p=0.032$ ), but not in women (OR 0.95, CI 95\% $0.87-1.03, p=0.218$ ). BMI, current smoking, alcohol consumption, and living alone did not affect the chance of PIM use (Table 1). This means that every year of additional education reduced the probability of taking PIMs by $10 \%$, at least in men.

Associations of PIM Use with Functional Assessments in the Overall Study Population and Stratified by Sex

We hypothesized PIM use to be associated with impaired physical and mental function. Thus, we examined the odds of PIM use with the results of functional geriatric assessments. Online supplementary Table 5 summarizes the assessment results related to PIM use in the overall study population.

Falls (OR 1.53, CI 95\% 1.08-2.17, $p=0.016$ ), frailty/ prefrailty (OR 1.68, 1.17-2.41, $p=0.005$ ), and depression (OR 2.12, CI 95\% 1.32-3.41, $p=0.002$ ) were associated with increased odds of PIM use. A better nutritional status was associated with lower odds of PIM use (OR 0.88, CI 95\% 0.81-0.97, $p=0.008)$. Results in the TUG test, the walking speed test $(4 \mathrm{~m})$, maximum handgrip strength, MMSE, DSST, and the clock drawing test were not associated with the odds of PIM use in the overall study population (online suppl. Table 5).

Sex-stratified analyses revealed that being frail/prefrail was associated with a higher prevalence of PIM use in men (OR 2.04, CI 95\% 1.18-3.54, $p=0.011)$. A better nutritional status was associated with a lower chance of PIM use in men (OR 0.83, CI 95\% 0.72-0.96, $p=0.011$ ). Falling in the past 12 months was associated with an increased odds of PIM use in women (OR 1.74, CI 95\% 1.10-2.75, $p=0.019$ ), while depression increased the odds of PIM use in both men (OR 2.74, CI 95\% 1.20-6.24, $p=0.016$ ) and women (OR 2.06, CI 95\% 1.14-3.71, $p=0.017$ ). Results in the TUG test, the walking speed test $(4 \mathrm{~m})$, maximum handgrip strength, MMSE, DSST, and the clock 
Table 2. Sex-stratified results of multivariable logistic regressions of functional assessments related to PIM use

\begin{tabular}{lllll}
\hline Assessment & OR & CI 95\% & $N$ & $p$ value \\
\hline Men & & & & \\
TUG & 1.03 & $0.89-1.18$ & 451 & 0.721 \\
Walking speed test, 4 m & 1.03 & $0.62-1.72$ & 230 & 0.901 \\
Handgrip strength, max. & 1.00 & $0.96-1.04$ & 458 & 0.967 \\
Falls in the past 12 months & 1.37 & $0.79-2.36$ & 449 & 0.259 \\
Fried frailty score & 2.04 & $1.18-3.54$ & 429 & $0.011^{*}$ \\
MNA & 0.83 & $0.72-0.96$ & 443 & $0.011^{*}$ \\
MMSE & 1.09 & $0.89-1.33$ & 449 & 0.396 \\
DSST & 1.01 & $0.98-1.05$ & 400 & 0.468 \\
Clock drawing test (Shulman) & 1.14 & $0.67-1.95$ & 434 & 0.631 \\
CES-D scale & 2.74 & $1.20-6.24$ & 450 & $0.016^{*}$ \\
Women & & & & \\
TUG & 1.00 & $0.87-1.14$ & 492 & 0.982 \\
Walking speed test, 4 m & 0.90 & $0.57-1.42$ & 159 & 0.653 \\
Handgrip strength, max. & 0.99 & $0.95-1.04$ & 495 & 0.756 \\
Falls in the past 12 months & 1.74 & $1.10-2.75$ & 479 & $0.019^{*}$ \\
Fried frailty score & 1.55 & $0.96-2.51$ & 462 & 0.073 \\
MNA & 0.93 & $0.82-1.04$ & 489 & 0.206 \\
MMSE & 1.17 & $0.96-1.44$ & 491 & 0.125 \\
DSST & 1.00 & $0.97-1.03$ & 422 & 0.984 \\
Clock drawing test (Shulman) & 1.17 & $0.74-1.86$ & 477 & 0.497 \\
CES-D scale & 2.06 & $1.14-3.71$ & 484 & $0.017^{*}$ \\
\hline
\end{tabular}

Logistic regressions were performed separately for each assessment including age, number of regular medications, morbidity index, and years of education as covariates. Detailed information on the functional assessments is available in the online supplementary material. CES-D Scale, Center for Epidemiological Studies Depression Scale; OR, odds ratio; $\mathrm{CI}$, confidence interval; PIM, potentially inappropriate medication; MNA, Mini-Nutritional Assessment; MMSE, Mini-Mental State Examination; DSST, digit-symbol substitution test; TUG, timed “up \& go.” * $p<0.05$. drawing test did not relate to the chance of PIM use (Table 2).

\section{Sex Differences in PIM Use regarding Drug Classes}

Since we detected sex differences regarding associations of PIM use with education and frailty/prefrailty, nutritional status and falls, we hypothesized that different patterns of PIM use in men and women may be responsible for the observations. We thus examined the distribution of PIMs used by men and women within different drug classes (Table 3 ). Online supplementary Table 6 presents the sex-stratified distribution of all PIM substances.

More men than women used PIMs of the ATC-group cardiovascular system $(p=0.036)$ and more women than men used PIMs of the ATC-group Genitourinary system and sex hormones $(p<0.001)$. Within the other groups analyzed, no differences in distribution frequencies were detected between sexes. For the group blood and blood forming organs, no test was performed due to the insufficient number of cases. Above that, the proportion of drugs with anticholinergic effects was similar in both sexes and showed no significant difference $(p=0.471)$.

\section{Discussion}

\section{Sex Differences in Participants Characteristics Related} to PIM Use

We found an association between higher education and lower prevalence of PIM use, which was only confirmed for men in the sex-stratified analysis. Male participants reported more years of formal schooling than female participants in the BASE-II cohort. This is in line with previous research, such as the nationally representative NAKO Study (German name: "Nationale Kohorte"), which identified men to have a higher education level than women [33]. Although the mean age 
Table 3. PIM use in men and women stratified by ATC-groups

\begin{tabular}{|c|c|c|c|c|}
\hline ATC group & $\begin{array}{l}\text { PIMs used by men } \\
(N=166) \\
(\Sigma 100 \%), n(\%)\end{array}$ & $\begin{array}{l}\text { PIMs used by women } \\
(N=236) \\
(\Sigma 100 \%), n(\%)\end{array}$ & $p$ value & $\begin{array}{l}\text { PIMs overall } \\
(N=402) \\
(\Sigma 100 \%), n(\%)\end{array}$ \\
\hline Blood and blood forming organs & $4(2.4)$ & $2(0.8)$ & - & $6(1.5)$ \\
\hline Cardiovascular system & $34(20.5)$ & $30(12.7)$ & $0.036^{*}$ & $64(15.9)$ \\
\hline Genitourinary system, sex hormones & $10(6.0)$ & $46(19.5)$ & $<0.001^{*}$ & $56(13.9)$ \\
\hline Respiratory system & $5(3.0)$ & $8(3.4)$ & 0.833 & $13(3.2)$ \\
\hline Thereof substances with anticholinergic effects each in men, women, and overall & $27(16.3)$ & $45(19.1)$ & 0.471 & $72(17.9)$ \\
\hline
\end{tabular}

PIMs classified by ATC classification system 1st level group and numbers of PIMs used by men and women. ATC, Anatomical Therapeutic Chemical; PIMs, potentially inappropriate medications. ${ }^{*} p<0.05$.

of participants in the NAKO cohort study is lower than the participants studied here ( 52 years vs. 69 years), the trend in terms of sex differences regarding years in schooling is consistent. Concerning the living situation, more participants in the cohort studied here live alone than the participants of the NAKO cohort study $(39.1 \%$ vs. $19.8 \%)$, that is possibly related to the older age of BASE-II participants. The participants BMI did not affect the chance of PIM use in our study. In contrast, the ESTHER study identified a higher BMI as a proxy for obesity to be a risk factor for PIM use in a cross-sectional analysis, but not in a longitudinal analysis [9]. A study investigating the relation between PIM use and "unhealthy behaviors" such as active smoking or heavy alcohol consumption found these factors to increase the chance of PIM use in older patients in primary care [13]. In our study, current smoking, alcohol consumption and living alone did not affect the chance of PIM use. This indicates that education affects the odds of PIM use stronger than lifestyle factors in communitydwelling older adults. In accordance with our results, multiple studies reported that PIM users exhibit lower levels of education than non-PIM users [34-36] and identified low socioeconomic characteristics to reduce the probability of optimal pharmacotherapy [36]. Differing physicians prescribing behavior can lead to increased inappropriate drug use in lower educated patients but also a more critical attitude of higher educated patients toward medications is a possible explanation of our findings. In order to minimize ADEs, physicians should critically review their prescribing behavior to avoid discrimination based on patient's education.

Associations with Inappropriate

Medication Use in Older Participants

\section{Sex Differences in Associations of PIM Use with} Assessment Results

We found associations of PIM use with frailty/prefrailty, nutritional status, falls, and depression in the overall study population. These findings were confirmed for frailty/prefrailty, nutritional status, and depression in men and for falls and depression in women.

Being frail/prefrail and having a lower nutritional status was associated with a higher prevalence of PIM use in men. Indeed, a low nutritional status may lead to malnutrition sarcopenia resulting in weakness or unintended weight loss as constituents of the Fried Frailty status. About one-third of the BASE-II participants were frail or prefrail [27], whereby men and women were equally affected by frailty (online suppl. Table 7). A longitudinal cohort study investigating community-dwelling older adults since 2008 reported a prevalence of $9.1 \%$ for frailty applying the Fried frailty criteria and an incidence of $21.0 \%$ for frailty within an average follow-up time of 4.8 years [16]. In cross-sectional analyses, an association of PIM use with frailty was reported for the EU(7)-PIM list [2], the PRISCUS list [6], the Beers PIM list 2015 [17], and the Beers dementia sub-list (as part of the Beers list 2015) in regression models including age and sex [16]. Complex regression models of the latter study additionally including number of regular medications, school education, household income, smoking status, BMI, and a total comorbidity score identified only the Beers dementia sublist to be associated with frailty [16]. The Beers dementia sub-list includes antipsychotics, anticholinergics, benzodiazepines, and $z$-substances [17]. The association confirmed in the complex model points toward an association of PIM use with frailty, thereby limiting the associa- 
tion to drugs with strong anticholinergic properties [16]. In contrast to one-dimensional assessments, like walking speed or handgrip strength, the rather complex frailty/ prefrailty syndrome showed an association with PIM use. This emphasizes the importance of complex geriatric assessments in everyday clinical practice because they seem to be more suitable to detect negative drug effects among geriatric patients. Frailty assessments can serve as indicators to detect negative effects related to drug use, but they can also help to evaluate potential benefits from drug therapy, as lower evidence regarding pharmacotherapy in frail patients can lead to an overestimation of benefits from drug use in this patient group than in robust older adults [37].

We identified falls in the past 12 months to be associated with PIM use in women. The prevalence of PIM use is generally higher in women than men due to higher rates of depressive episodes and anxiety disorders [12], leading to a more frequent use of antidepressants, benzodiazepines, and $z$-substances. Especially some drugs of the ATC-group nervous system have anticholinergic side effects, thereby increasing the risk of falling $[38,39]$. Nevertheless, the proportion of male and female PIM users within the group Nervous system is almost equal in our cohort, which might indicate differing effects of anticholinergics in women compared to men. The association of anticholinergic drug burden with falling, which has been described in several studies $[38,39]$, is the reason for anticholinergics to be included in the EU(7)-PIM list, besides the risk of cognitive impairment [2]. Falling has to be thoroughly avoided in older adults because it may lead to fractures [40], which in turn are associated with loss of mobility and increased mortality [41]. Falls were reported more frequently overall by female study participants (online suppl. Table 7). It is possible that women actually fell more often, but also a bias in reporting falls is conceivable. This could also be a reason for the lack of association between PIM use with falls in men, despite the balanced distribution of PIMs in men and women within the group Nervous system and the detected association of PIM use with frailty in men, which has already been described to be related to anticholinergic drug use [16].

The association of PIM use and depression was confirmed in both sexes in separated regression analysis. Depression itself can be the reason for PIM prescription as many drugs classified as PIMs are antidepressants. Although depression, anxiety, and sleep disorders are more prevalent in women overall [12], we did not find a higher proportion of female PIM users in the ATC-group nervous system in the BASE-II cohort. This result seems con- clusive because there are no sex-dependent differences in treatment strategies in depression until now. Depression significantly increases the chance of PIM use in the study population, which is in line with results from other studies describing depressive episodes to be strongly associated with PIM use $[9,42]$. With respect to the variables considered, we identified the association between depression and PIM use as the most robust association in this study, as it was found both in the overall cohort and in the sex-stratified analysis. This finding emphasizes the importance of depression diagnosis and appropriate treatment, which can be more difficult in old age, especially in patients with comorbidities [43, 44]. In older patients, nonpharmacological treatments are underutilized as alternative strategies to drug therapy, even though psychotherapy is rated as particularly beneficial for older as well as for multimorbid patients $[1,43]$.

We did not find results of physical assessments applying the TUG test, walking speed test ( $4 \mathrm{~m})$, and maximum handgrip strength to be associated with PIM use. An association regarding functional impairment was described for the ESTHER cohort [9]. In contrast to the ESTHER cohort study, which identified cognitive impairment to be strongly associated with the use of PIMs in cross-sectional analysis [9], PIM use was not associated with reduced cognitive performance in our study applying the MMSE, DSST, and the Clock drawing test. The mean age of the participants was about 70 years in both, the ESTHER cohort study and the BASE-II cohort study [9]. Thus, age differences in the cohorts cannot explain the divergent findings, but BASE-II participants are above average education [20]. Possibly, negative PIM effects are compensated through education leading to the missing associations between PIM use and cognitive test results in our study.

\section{Sex Differences in PIM Use regarding Drug Classes}

We found significant sex-dependent distribution differences in two drug classes. Although cardiovascular diseases occur likewise common in both sexes, more men than women used PIMs attributable to the cardiovascular system. Drugs belonging to this group are used to treat heart failure, hypertension, or cardiac arrythmia. More women than men used PIMs attributable to the genitourinary system and sex hormones. This can be explained by the common use of hormone replacement therapy in women. In contrast, anticholinergic drugs were used as often by men as by women. Although these were used equally in both men and women, we detected sex differences in the associations of PIM use with frailty/prefrail- 
ty and falling, which are both related to anticholinergic drug burden $[16,38-40]$. This finding might point toward differing effects of anticholinergic drug use in men apart from women.

\section{Strengths and Limitations}

To capture PIMs, we recorded prescribed drugs as well as nonprescribed drugs and dietary supplements within an interview. Information regarding participants' characteristics and functional capacity were recorded in a structured interview, except from the physical assessments, like walking speeds and handgrip strength. Consequently, the recorded results might be biased through reporting. Although a large cohort was investigated, study participants are characterized by above average education, health, and life satisfaction [45], so that underestimation of negative PIM effects is possible. Our results might not generalize to more vulnerable segments of the population. Furthermore, we found associations in cross-sectional analysis, but we cannot specify the direction of the associations found. PIM use might affect assessment results, but causality is uncertain, due to the cross-sectional approach.

\section{Conclusion}

While education as a socioeconomic indicator predicted the prevalence of PIM use in men, lifestyle factors investigated, such as smoking or living alone did not. PIM use appeared to be associated with frailty in men, while it was associated with falling in the last 12 months in women. Further studies are needed to examine whether differing associations of PIM use in men and women are attributable to sex-dependent, variable effects of the anticholinergic drug burden of PIMs.

\section{Acknowledgments}

The authors are grateful to the participants of this study.

\section{Statement of Ethics}

All participants gave written informed consent, and the Ethics Committee of the Charité-Universitätsmedizin Berlin approved the BASE-II study (approval number EA2/029/09).

\section{Conflict of Interest Statement}

The authors declare that they have no conflicts of interest.

\section{Funding Sources}

The BASE-II research project (Co-PIs are Lars Bertram, Ilja Demuth, Denis Gerstorf, Ulman Lindenberger, Graham Pawelec, Elisabeth Steinhagen-Thiessen, and Gert G. Wagner) is supported by the German Federal Ministry of Education and Research (Bundesministerium für Bildung und Forschung, BMBF) under grant numbers \#16SV5536K, \#16SV5537, \#16SV5538, \#16SV5837, \#01UW0808, 01GL1716A, and 01GL1716B. Another source of funding is the Max Planck Institute for Human Development, Berlin, Germany. Additional contributions (e.g., equipment, logistics, and personnel) are made from each of the other participating sites. The funders had no role in study design, data collection and analysis, decision to publish, or preparation of the manuscript.

\section{Author Contributions}

Juliane Bolbrinker, Ilja Demuth, Reinhold Kreutz, and Sarah Toepfer contributed to conceptualization. Sarah Toepfer contributed to formal analysis and writing - original draft. Project administration was carried out by Ilja Demuth. Supervision was done by Juliane Bolbrinker and Ilja Demuth. Juliane Bolbrinker, Ilja Demuth, Johanna Drewelies, Maximilian König, Reinhold Kreutz, and Dominik Spira contributed to writing - review and editing.

\section{Data Availability Statement}

Due to concerns for participant privacy, data are available only upon request. Data access is restricted by the BASE-II Steering Committee. External scientists may apply to the Steering Committee for data access. Please contact Ludmila Müller, scientific coordinator, atlmueller@mpib-berlin.mpg.de.

\section{References}

1 Fialova D, Laffon B, Marinkovic V, Tasic L, Doro P, Somicronos G, et al. Medication use in older patients and age-blind approach: narrative literature review (insufficient evidence on the efficacy and safety of drugs in older age, frequent use of PIMs and polypharmacy, and underuse of highly beneficial nonpharmacological strategies). Eur J Clin Pharmacol. 2019;75(4):451-66.
2 Renom-Guiteras A, Meyer G, Thürmann PA The EU (7)-PIM list: a list of potentially inappropriate medications for older people consented by experts from seven European countries. Eur J Clin Pharmacol. 2015;71(7):86175.

3 Novaes PH, da Cruz DT, Lucchetti ALG, Leite ICG, Lucchetti G. Comparison of four criteria for potentially inappropriate medications in Brazilian community-dwelling older adults. Geriatr Gerontol Int. 2017;17(10):1628-35.
Associations with Inappropriate

Medication Use in Older Participants 
4 Motter FR, Fritzen JS, Hilmer SN, Paniz ÉV, Paniz VMV. Potentially inappropriate medication in the elderly: a systematic review of validated explicit criteria. Eur J Clin Pharmacol. 2018;74(6):679-700.

5 Fialova D, Brkic J, Laffon B, Reissigova J, Gresakova S, Dogan S, et al. Applicability of EU (7)-PIM criteria in cross-national studies in European countries. Ther Adv Drug Saf. 2019;10:2042098619854014.

6 Holt S, Schmiedl S, Thürmann PA. Potentially inappropriate medications in the elderly: the PRISCUS list. Dtsch Arztebl Int. 2010; 107(31-32):543-51.

7 Toepfer S, Bolbrinker J, König M, SteinhagenThiessen E, Kreutz R, Demuth I. Potentially inappropriate medication in older participants of the berlin aging study II (BASE-II) - Sex differences and associations with morbidity and medication use. PLoS One. 2019; 14(12):e0226511.

8 Morgan SG, Weymann D, Pratt B, Smolina K, Gladstone EJ, Raymond C, et al. Sex differences in the risk of receiving potentially inappropriate prescriptions among older adults. Age Ageing. 2016;45(4):535-42.

9 Muhlack DC, Hoppe LK, Stock C, Haefeli WE, Brenner H, Schöttker B. The associations of geriatric syndromes and other patient characteristics with the current and future use of potentially inappropriate medications in a large cohort study. Eur J Clin Pharmacol. 2018;74(12):1633-44.

10 Johnell K, Weitoft GR, Fastbom J. Sex differences in inappropriate drug use: a registerbased study of over 600,000 older people. Ann Pharmacother. 2009;43(7):1233-8.

11 Johnell K, Fastbom J. Gender and use of hypnotics or sedatives in old age: a nationwide register-based study. Int J Clin Pharm. 2011; 33(5):788-93.

12 Koch-Institut R. Gesundheit in Deutschland: Robert Koch-Institut; 2015. p. 516.

13 Projovic I, Vukadinovic D, Milovanovic O, Jurisevic M, Pavlovic R, Jacovic S, et al. Risk factors for potentially inappropriate prescribing to older patients in primary care. Eur J Clin Pharmacol. 2016;72(1):93-107.

14 Nothelle SK, Sharma R, Oakes A, Jackson M, Segal JB. Factors associated with potentially inappropriate medication use in communitydwelling older adults in the United States: a systematic review. Int J Pharm Pract. 2019.

15 Selke Krulichova I, Selke GW, Thurmann PA. Trends and patterns in EU (7)-PIM prescribing to elderly patients in Germany. Eur J Clin Pharmacol. 2021.

16 Muhlack DC, Hoppe LK, Saum KU, Haefeli WE, Brenner H, Schöttker B. Investigation of a possible association of potentially inappropriate medication for older adults and frailty in a prospective cohort study from Germany. Age Ageing. 2019;49(1):20-5.

17 By the American Geriatrics Society Beers Criteria Update Expert Panel. American geriatrics society 2015 updated beers criteria for potentially inappropriate medication use in old- er adults. J Am Geriatr Soc. 2015;63(11): 2227-46.

18 Whayne TF Jr. Clinical use of digitalis: a state of the art review. Am J Cardiovasc Drugs. 2018;18(6):427-40.

19 (DIMDI) DIfMDuI. Anatomisch-therapeutisch-chemische-Klassifikation mit Tagesdosen Amtliche Fassung des ATC-Index mit DDDAngaben für Deutschland im Jahre; 2020.

20 Bertram L, Böckenhoff A, Demuth I, Düzel S, Eckardt R, Li SC, et al. Cohort profile: the berlin aging study II (BASE-II). Int J Epidemiol. 2014;43(3):703-12.

21 Gerstorf D, Bertram L, Lindenberger U, Pawelec G, Demuth I, Steinhagen-Thiessen E, et al. Behavioural science section/the Berlin aging study II: an overview. Gerontology. 2016;62(3):311-5.

22 Charlson ME, Pompei P, Ales KL, MacKenzie $\mathrm{CR}$. A new method of classifying prognostic comorbidity in longitudinal studies: development and validation. J Chronic Dis. 1987; 40(5):373-83.

23 Meyer A, Salewsky B, Spira D, SteinhagenThiessen E, Norman K, Demuth I. Leukocyte telomere length is related to appendicular lean mass: cross-sectional data from the Berlin aging study II (BASE-II). Am J Clin Nutr. 2016;103(1):178-83.

24 Mahoney FI, Barthel DW. Functional evaluation: the barthel index. Md State Med J. 1965; 14:61-5.

25 Lawton MP, Brody EM. Assessment of older people: self-maintaining and instrumental activities of daily living. Gerontologist. 1969; 9(3):179-86.

26 Fried LP, Tangen CM, Walston J, Newman $\mathrm{AB}$, Hirsch C, Gottdiener J, et al. Frailty in older adults: evidence for a phenotype. J Gerontol A Biol Sci Med Sci. 2001;56(3): M146-56.

27 Spira D, Buchmann N, Nikolov J, Demuth I, Steinhagen-Thiessen E, Eckardt R, et al. Association of low lean mass with frailty and physical performance: a comparison between two operational definitions of sarcopenia-data from the Berlin aging study II (BASE-II). J Gerontol A Biol Sci Med Sci. 2015;70(6):779-84.

28 Vellas B, Guigoz Y, Garry PJ, Nourhashemi F, Bennahum D, Lauque $S$, et al. The mini nutritional assessment (MNA) and its use in grading the nutritional state of elderly patients. Nutrition. 1999;15(2):116-22.

29 Folstein MF, Folstein SE, McHugh PR. Minimental state. A practical method for grading the cognitive state of patients for the clinician. J Psychiatr Res. 1975;12(3):189-98.

30 Jaeger J. Digit symbol substitution test: the case for sensitivity over specificity in neuropsychological testing. J Clin Psychopharmacol. 2018;38(5):513-9.

31 Shulman KI. Clock-drawing: is it the ideal cognitive screening test? Int J Geriatr Psychiatry. 2000;15(6):548-61.

32 Radloff LS. The CES-D scale: a self-report depression scale for research in the general population. Appl Psychol Meas. 1977:385-401.
33 Dragano N, Reuter M, Greiser KH, Becher H, Zeeb H, Mikolajczyk R, et al. Socio-demographic and employment-related factors in the German National Cohort (GNC; NAKO Gesundheitsstudie). Bundesgesundheitsblatt Gesundheitsforschung Gesundheitsschutz. 2020;63(3):267-78.

34 Weston AL, Weinstein AM, Barton C, Yaffe K. Potentially inappropriate medication use in older adults with mild cognitive impairment. J Gerontol A Biol Sci Med Sci. 2010; 65(3):318-21.

35 Haider SI, Johnell K, Weitoft GR, Thorslund $\mathrm{M}$, Fastbom J. The influence of educational level on polypharmacy and inappropriate drug use: a register-based study of more than 600,000 older people. J Am Geriatr Soc. 2009; 57(1):62-9.

36 Lechevallier-Michel N, Gautier-Bertrand M, Alpérovitch A, Berr C, Belmin J, Legrain S, et al. Frequency and risk factors of potentially inappropriate medication use in a community-dwelling elderly population: results from the 3C Study. Eur J Clin Pharmacol. 2005; 60(11):813-9.

37 Liau SJ, Lalic S, Sluggett JK, Cesari M, Onder G, Vetrano DL, et al. Medication management in frail older people: consensus principles for clinical practice, research, and education. J Am Med Dir Assoc. 2021;22(1): 43-9.

38 Green AR, Reifler LM, Bayliss EA, Weffald LA, Boyd CM. Drugs contributing to anticholinergic burden and risk of fall or fall-related injury among older adults with mild cognitive impairment, dementia and multiple chronic conditions: a retrospective cohort study. Drugs Aging. 2019;36(3):289-97.

39 Byrne CJ, Walsh C, Cahir C, Bennett K. Impact of drug burden index on adverse health outcomes in Irish community-dwelling older people: a cohort study. BMC Geriatr. 2019; 19(1):121.

40 Suehs BT, Caplan EO, Hayden J, Ng DB, Gaddy RR. The relationship between anticholinergic exposure and falls, fractures, and mortality in patients with overactive bladder. Drugs Aging. 2019;36(10):957-67.

41 Pahor M. Falls in older adults: prevention, mortality, and costs. JAMA. 2019;321(21): 2080-1.

42 Kucukdagli P, Bahat G, Bay I, Kilic C, Oren MM, Turkmen BO, et al. The relationship between common geriatric syndromes and potentially inappropriate medication use among older adults. Aging Clin Exp Res. 2019.

43 Stanners MN, Barton CA, Shakib S, Winefield HR. Depression diagnosis and treatment amongst multimorbid patients: a thematic analysis. BMC Fam Pract. 2014;15:124

44 Mitchell PB, Harvey SB. Depression and the older medical patient when and how to intervene. Maturitas. 2014;79(2):153-9.

45 Bertram L, Böckenhoff A, Demuth I, Düzel S, Eckardt R, Li SC, et al. Cohort profile: the Berlin aging study II (BASE-II). Int J Epidemiol. 2014;43(3):703-12. 